\title{
Quantitative Analysis of White Matter Fiber Properties along Geodesic Paths
}

\author{
Pierre Fillard ${ }^{1,4}$, John Gilmore ${ }^{2}$, Joseph Piven ${ }^{2}$, Weili Lin ${ }^{3}$, and Guido Gerig ${ }^{1,2}$ \\ 1 Department of Computer Science, \\ 2 Department of Psychiatry, \\ 3 Department of Radiology, \\ University of North Carolina, Chapel Hill, NC 27599, USA \\ 4 ESCPE Lyon, 69100 Villeurbanne, FRANCE, \\ \{fillard,gerig\}@cs.unc.edu \\ Software Download: http://midag.cs.unc.edu ${ }^{\ddagger}$
}

\begin{abstract}
Diffusion Tensor Imaging (DTI) is becoming a routine magnetic resonance technique to study white matter properties and alterations of fiber integrity due to pathology. The advanced MRI technique needs postprocessing by adequate image processing and visualization tools. Analysis of DTI in clinical studies so far use manual definition of regions or interest or image matching followed by voxel-based analysis. This paper presents a novel concept that extracts major fiber bundles by tractography and provides a statistical analysis of diffusion properties along fibers, i.e. geodesic paths within the three-dimensional brain image. Fiber tracing thus serves as a sophisticated, efficient method for defining complex regions of interests along major fiber tracts not accessible otherwise. Fiber bundles extracted from a set of subjects are parametrized by arc-length and mapped to a common coordinate system centered at well-defined anatomical landmarks. The description of the methodology is guided by the example of measuring diffusion properties along the left and right cingulate. We also present preliminary results from an ongoing clinical neonatal study that studies early brain development.
\end{abstract}

\section{Introduction}

Diffusion Tensor Imaging (DTI) is an MRI technique which assesses brain tissue properties via diffusivity, initiated by seminal research by Basser, Pierpaoli and others [1,2]. Water shows restricted diffusivity as a function of material properties, and it tends to show stronger diffusion along oriented tissue such as white matter fibers which is inhibited orthogonally. Therefore, extraction of

\footnotetext{
$\ddagger$ This research is supported by the UNC Neurodevelopmental sorders Research Center HD 03110, the NIH Conte Center MH064065, the Stanley Medical Research Institute, and the Foundation of Hope (Raleigh, NC).We are greatful to Ch. Davatzikos, D. Xu, D. Shen (all University of Pennsylvania), and S. Mori, Johns Hopkins University, for providing an early version of the fiber-tracking tool. A color paper version if found at: www.cs.unc.edu/ /gerig.
} 
local diffusion tensors via MRI and measurements derived from these tensors have become powerful techniques to study the local structure of intra-cellular and extracellular space within brain tissue.

Alterations of the density of axons or degree of myelination, and occurrence of lesions and infiltrating tumors, for example, have strong effects on the magnitude and shape of local diffusion tensors. The study of local properties of DTI have thus become a standard tool to study white matter disease. Beyond strictly local properties, white matter is subdivided into fiber tracts based on anatomical and functional criteria. These fiber bundles are assessed in-vivo by techniques commonly called tractography. The vector-field defined by the set of longest tensor axes is traced between source and target regions. This results in curvilinear paths which most likely represent elements of fiber bundles. Research in MRI and Psychiatry even goes a step further and tries to establish a link between diffusion properties of fiber tracts and brain connectivity. This could potentially explain changes in functional connectivity as measured by fMRI and lead to a better understanding of neurodevelopmental or neurodegenerative brain changes.

Clinical studies using DTI most often rely on comparison of regions-of-interest (ROIs) specified by users [3] or on voxel-based analysis of registered image datasets 4. Whereas the former is inherently limited by the problem of reliable specification of small ROIs across a large number of 3-D images, the latter requires sophisticated registration and postprocessing techniques to account for local distortion of DTI obtained via echo-planar imaging (EPI), for warping individual brain structures to a template, and to solve tensor re-orientation as part of the non-linear warping [5]. Herein, we propose an alternative processing scheme. We use tractography to extract sought fiber tracts from a series of image data. Fiber tracts are stored as sets of curvilinear structures with subvoxel precision and are parametrized by arc-length. These bundles are mapped into into a graph after centering at a common origin defined by a well-defined anatomical landmark. Statistical analysis of diffusion tensors thus can be performed along tracts, which are geodesic paths in the original image data. Section 3 presents early results from an ongoing clinical study of early brain development. Commissural tracts through splenium and genu of the corpus callosum of newborns ( 2 weeks old) are compared to those extracted from 2-years old children and to adults.

\section{Extraction of Fiber Tracts}

We have combined common, well-established processing methods for DTI data, a recently developed powerful fiber tracking method and interactive scientific visualization into an integrated tool. The tool is integrated into ITK, openplatform, and freely available at midag.cs.unc.edu.

Tensor Coefficients Calculation. The tensor field calculation is based on an analytical solution of the Stejskal and Tanner's diffusion equation system (a comprehensive description is found in [6]). The current version is designed to 
use the common directional coding proposed by Basser et al. 2]. The baseline and six directional images are loaded into the program for extraction of the diffusion tensor. The two measures most commonly used in clinical analysis, the "apparent diffusion coefficient" (ADC, trace of tensor) and the "fractional anisotropy" (FA, shape described by tensor), are calculated and can be stored as image data. Non-brain structures are suppressed by a user-defined threshold on the diffusion baseline image. All the images including the original DTI data and tensor measurement can be selected for multi-planar visualization.

Fiber-Tracking Algorithm. The vector field defined by the eigenvectors associated with the largest eigenvalues is assumed to represent a good approximation to local white matter fiber orientation. This simplification fails at branchings and crossings of nearby fiber bundles, which is subject to ongoing research by MR researchers using a larger number of discrete directions or advanced techniques like diffusion spectrum imaging (DSI). The goal of the tracking, also called "tractography", is to find likely paths through the vector field between source and target regions of interest (ROIs). We reimplemented a previously published method [5].

Extensions. We apply the tracking method with a backward tracking scheme instead of a direct tracking. Direct tracking is a forward processing scheme which has the disadvantages that it can provide only one trace per voxel and that it has to make local decisions for path propagation. The backward scheme as used herein is initialized at each voxel of the full brain (target). To reduce computational effort, only voxels above a user-specified minimal FA value are considered for tracing. The method traces paths from each target voxel backwards to the source region, and only fiber tracings passing through the user selected ROIs are finally kept. This concept, assuming that the target volume is much larger than the source ROI, makes use of the decrease of the complexity while propagating and results in a significantly improved robustness.

Figure 1 illustrates the reconstruction of the left and right cingulate fiber tracts in an adult case. The ROIs for the tracking (defined with IRIS/SNAP) are placed in the anterior and posterior regions of the cingulate.

\subsection{Mapping of Fibers to Corresponding Coordinate Frame}

Fiber bundles traced between target and source are stored as sets of curvilinear poly-lines using the standardized data structure provided by ITK [7]. Within each bundle, the coordinate origin is specified as a key anatomical landmark specified by the user. The specification of such an origin might vary for various types of fiber tracts. Commissural tracts through the corpus callosum, for example, use the midsagittal plane coordinate as the common center. The cingulate, on the other hand, uses the specification of the center of the nearby splenium as an external anatomical landmark to represent the most inferior location for left and right structures. Concepts for aligning fiber bundles using 3-D curve matching strategies were presented by Ding et al. [8]. 
The curvilinear structures carry FA and ADC values as attributes at each node. This list of attributes can be easily extended upon user's needs, e.g by combining values extracted from structural images. It is straightforward to combine the FA values of the set of single fibers to an average $\overline{F A}$ and to calculate statistics as a function of distance from the origin $F A(d)$ :

$F A_{i}(d) \mid d=\left\{-d_{\min }, \cdots, 0, \cdots, d_{\max }\right\}$ and $\overline{F A}(d)=\frac{1}{n} \sum_{i=1}^{n} F A_{i}(d)$.

The same concept is applied to combine fiber tracts among different individuals, assuming that the tracked structures represent comparable anatomical regions. Please note that this mapping represents a geodesic path in the individual datasets as shown in Figure 1 middle. Fig. 1 right illustrates the FA values for the left and right cingulate fiber tracts as functions of geodesic distance along the tracts. Left to right shows posterior to anterior direction.
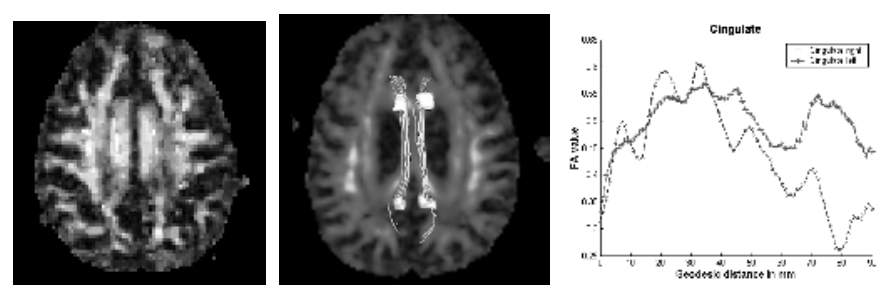

Fig. 1. Reconstruction of the cingulate tract. Left: Color-coded FA image at the level of the left and right cingulate (anterior-posterior, inferior-superior and left-right orientations). Middle: Result of the fiber tracking with source and target regions for initializing the tracking (blobs). Right: FA values for left and right cingulate plotted as a function of geodesic distance, left to right represents posterior to anterior.

\section{Application to Neonate Study}

While it has been hypothesized that brain development is abnormal in neurodevelopmental disorders, there have been very few attempts to study very early brain development in children. Only a few prior quantitative magnetic resonance imaging and diffusion tensor imaging studies of unsedated newborns exist; most of the children in these studies were born prematurely 91011.

In a pilot study to optimize procedures for a much larger full-scale clinical study ([3]), 20 unsedated healthy newborns underwent 3 Tesla magnetic resonance imaging (MRI and DTI, 10 males and 10 females; age $16 \pm 4$ days [mean \pm $\mathrm{SD}]$ ). Scans without significant motion were obtained in 13 children. The imaging parameters for the DTI sequence were: TR/TE/TH $=4219 \mathrm{~ms} / 92.2 \mathrm{~ms} / 5 \mathrm{~mm}$, inplane resolution $=1.72 \times 1.72 \mathrm{~mm}, 12$ averages, and 20 slices. Seven images were acquired for each slice using the directional encoding scheme developed by Basser et al. 2]. Due to the pilot character of this study also including scanner pulse sequence optimization, we selected all 13 scans for ROI analysis in one axial slice (see subsection [3), but only a subset of five neonates for the more advanced fiber tracking method (subsection 3). 
Quantitative ROI analysis of DTI. Eight regions of interest (ROIs) were placed in white matter on a single transverse section through the level of the basal ganglia, including the anterior and posterior limbs of the internal capsule (IC), left and right occipital and frontal WM adjacent to the cortical gray matter and the genu and splenium of the corpus callosum [3]. Group comparisons showed a global elevation of ADC $(\mathrm{p}<0.001)$ in both GM and WM and a reduction of FA $(\mathrm{p}<0.001)$ in WM were found in neonates when compared with that in adults. While regional variations of FA and ADC in adults are less remarkable, the central WM in neonates consistently exhibits higher FA and lower ADC than peripheral WM.

Fiber tract analysis along geodesic paths. The ROI-based study discussed above is limited to measurements in only one axial cross-section. Further, reliable identification of ROIs at corresponding anatomical regions across subjects is a real challenge, especially in view of the low contrast-to-noise ratio in neonatal imagery. We applied our novel, highly automated measurement scheme to assess white matter properties in full 3-D space and tested the hypothesis of FA decrease along interior to peripheral directions. The user interaction is limited to the specification of splenium and genu of the corpus callosum, used as source regions for fiber tracking. This task can be reliably and efficiently by loading the FA image into our interactive image editing tool IRIS/SNAP (free download at midag.cs.unc.edu), which allows a user to define 3D regions of arbitrary shape, e.g. spheres, in volumetric image data. Fiber tracts were traced backwards from the full brain towards these source regions. Figures 2 represent 5 cases with commissural tracts through the splenium region and qualitatively demonstrates the reproducibility across subjects. Similar results were obtained for the tracts through the genu.
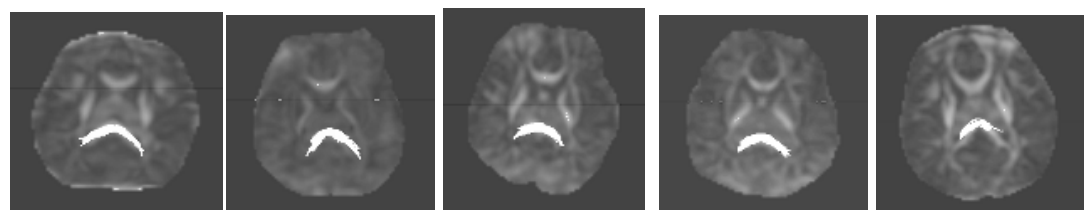

Fig. 2. Tracing of the commissural fiber tracts through the splenium of the corpus callosum shown in five neonates. The splenium is specified as a sphere centered at the midsagittal plane. The whole brain with FA larger than 0.1 is specified as the target region. Fibers are traced backwards from target to source.

Mapping of fibers into corresponding coordinate frame. The quantitative analysis of fiber tracts follows the procedure outlined in previous section 2.1 The coordinate origin for each fiber bundle was set to the center of the genu and splenium regions. Figure 3 illustrates the FA properties of the commissural tracts through the genu (left) and splenium (right) for five neonates. Individual curves (textured) such as average curve (solid blue) are shown in overlay. All curves cover a common distance of $p m 20 \mathrm{~mm}$ from the origin and clearly show a sharp decrease 
of FA with increasing distance from origin. Figure 4 illustrates the potential of our new method to address the important clinical question of maturation with age or alterations due to developmental delay. The genu and splenium tracking was additionally applied to DTI of healthy adults and 2-year old children. Each curve represents an average of several subjects. Given the small number, it would be too premature to draw final conclusions. However, the curves confirm earlier findings of smaller FA values in neonates 103. Interestingly, the 2-year old subjects seem to have values in the same range as adults.
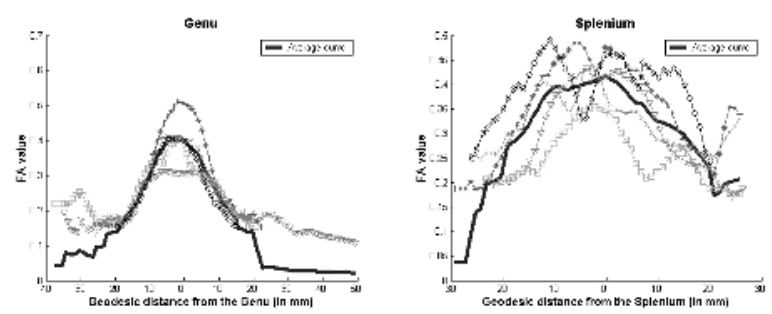

Fig. 3. Fractional anisotropy (FA) measured along commissural tracts through the genu (left) and splenium (right) shown for five neonates. The plots are centered at the position of the midsagittal plane and follow the fiber tracts exteriorly towards the cortex, mostly in anterior (genu) posterior (splenium) directions. The average $\overline{F A}$ curves are shown as a solid, blue lines.
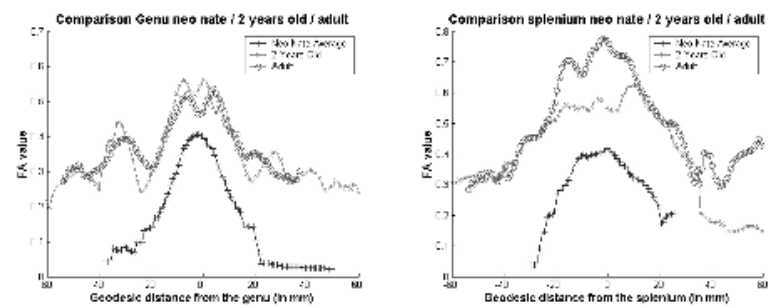

Fig. 4. Fractional anisotropy (FA) measured along commissural tracts through the genu (left) and splenium (right) as a function of age. The average of five neonates (blue) is compared to 2years old subjects (purple) and adults (red). The horizontal axis reflects real distance in $\mathrm{mm}$. Results suggest significantly smaller FA values for neonates and quick decrease towards peripheral regions.

Validation. In total, we tested the DTI analysis and fiber tracking tools on over 25 images taken from various ongoing clinical studies. Validation of DTI with comparison of different pulse sequences (EPI and spiral), scanner manufacturers (Siemens 3T, GE 1.5T), and spatial resolution is part of an ongoing activity between MRI research at UNC, Duke University and our lab. Validation of DTI fiber tracking requires simulated data, standardized phantoms and/or animal studies. Providing tools and methodology to assess validity is part of ongoing efforts of the MRI research community. 
Validation in our study was so far limited to assess reliability of the postprocessing methods. Systematic validation studies of user-defined ROI placement as described in paragraph 3 illustrated the problem of limited reliability and was key for the new development presented here. In this validation study with repeated measurements ( 5 cases with 3 repetitions each, 2 years old children), two manual raters blind to the data placed volumetric ROIs in frontal white matter as seen in ADC images, following a well-defined protocol based on anatomical landmarks. Statistical analysis of the apparent diffusion coefficient (ADC) and fractional anisotropy (FA) resulted in inter- and intra-rater reliabilies of 0.85 to 0.90, which was considered as insufficient. Our new tool method only requires the placement of two regions to cover the splenium and genu in the FA image. This simple task can be achieved with very high reliability. The remaining fiber tracking procedure with a fixed set of parameters is fully automatic and not subject to any user variability.

As part of the same study, we calculated an FA atlas of neonates by correcting for spatial distortions of DTI (warping DTI to corresponding T2 weighted spin-echo structural images), and aligning the individual subjects to a template defined in Talairach coordinate space. This resulting coordinate transformations will allow us to map the fiber tracts of individual subjects into a common coordinate frame and to assess inter-subject spatial variability.

\section{Discussion}

This report describes work in progress towards mapping and parametrizing fiber tracts extracted from a large number of subjects. We present a new approach for measuring white matter diffusion properties along fiber tracts as a function of geodesic distance from specific anatomical landmarks. Fiber tracking and alignment serve as new methods to establish homology among subjects. The procedure overcomes existing limitations of user-specified region definition or full-brain registration, especially in view of the spatial distortions faced with high-speed neonatal MRI. The tracking takes only a few seconds on a standard PC. The whole process including ROI specifications, FA calculation, tracking, and storing fibers into a database takes only a few minutes per subject. Current research addresses the issues of developing more advanced schemes for fiber tract alignment, and validation of fiber bundles obtained by tracking in comparison to co-registered structural image data.

Previous studies in infants and older children find that white matter FA increases and ADC decreases with age [9]101112]. In the very limited age range available to our study, we observed a significant increase in FA in the genu (r2 $=0.3639 ; \mathrm{p}=0.0291)$ and splenium $(\mathrm{r} 2=0.5102 ; \mathrm{p}=0.0091)$ of the corpus callosum, but not in other white regions. This suggests that the white matter of the corpus callosum is undergoing significant maturation in the period after birth that may represent a window of vulnerability to perinatal insults that have been associated with neurodevelopmental disorders.

This study is limited by the relatively small sample size and our findings need to be replicated in a larger sample. As part of our Conte Center to study 
pathophysiology in Schizophrenia, we expect to scan over 100 neonates, some with follow-up scans, over the next 5 years. As part of the activities of the neurodevelopmental research center, we will also apply our new DTI analysis technique to $2-4$ years old children (autistic subjects and controls, $\mathrm{N}=20+50$ ) and neonates $(\mathrm{N}=60+60)$. Further, we will explore other major fiber tracts known for delayed myelination, e.g. the temporal lobe connections.

\section{References}

1. P.J. Basser, J. Mattiello, and D. Le Bihan, "Estimation of the effective self-diffusion tensor from the nmr spin echo," Journal of Magnetic Resonance in Imaging JMRI, vol. 103, 1994.

2. P.J. Basser and C. Pierpaoli, "Microstructural and physiological features of tissues elucidated by quantitative diffusion tensor mri," Journal of Magnetic Resonance Imaging (JMRI), vol. 111, pp. 209-219, 1996.

3. G. Zhai, Weili Lin, K. Wilber, G. Gerig, and J.H. Gilmore, "Comparison of regional white matter diffusion in healthy neonates and adults using a 3t head-only $\mathrm{mr}$ scanner," Radiology, 2003, in print.

4. K.O. Lim, M. Hedehus, M. Moseley, A. De Crespigny, E.V. Sullivan, and A. Pfefferbaum, "Compromised white matter tract integrity in schizophrenia inferred from diffusion tensor imaging," Arch Gen Psychiatry, vol. 56, pp. 367-374, 1999.

5. D. Xu, S. Mori, M. Solaiyappan, P. C. M. van Zijl, and Ch. Davatzikos, "A framework for callosal fiber distribution analysis," NeuroImage, vol. 17, pp. 1131$1143,2002$.

6. C.F. Westin, S.E. Maier, H. Mamata, A. Nabavi, F.A. Jolesz, and R. Kikinis, "Processing and visulaization for diffusion tensor mri," Medical Image Analysis, vol. 6, pp. 93-108, 2002.

7. National Library of Medicine NLM, "Insight Toolkit ITK," 2002, http://www.itk.org.

8. Z. Ding, J.C. Gore, and A. Anderson, "Case study: Reconstruction, visualization, and quantification of neuronal fiber pathways," in Proceedings IEEE Visualization. IEEE, 2001, pp. 453-456.

9. P.S. Hüppi, S.E. Maier, S. Peled, G.P. Zientara, P.D. Barnes, F.A. Jolesz, and J.J. Volpe, "Microstructural development of human newborn cerebral white matter assessed in vivo by diffusion tensor magnetic resonance imaging," Pediatrics, vol. 44, pp. 584-590, 1998.

10. P.S. Hüppi, S. Warfield, R. Kikinis, P.D. Barnes, G.P. Zientara, F.A. Jolesz, M.K. Tsuji, and J.J. Volpe, "Quantitative magnetic resonance imaging of brain development in premature and normal newborns," Ann Neurol, vol. 43, pp. 224-235, 1998.

11. J.J. Neil, S.I. Shiran, R.C. McKinstry, G.L. Schefft, A.Z. Snyder, C.R. Almli, E. Akbudak, J.A. Aronovitz, J.P. Miller, B.C.P. Lee, and T.E. Conturo, "Normal brain in human newborns: apparent diffusion coefficient and diffusion anisotropy measured by using diffusion tensor mr imaging," Radiology, vol. 209, pp. 57-66, 1998.

12. V.J. Schmithorst, M. Wilke, B.J. Dardzinski, and S.K. Holland, "Correlation of white matter diffusivity and anisotropy with age during childhood and adolescence: a cross-sectional diffusion-tensor mr imaging study," Radiology, vol. 222, pp. 121$218,2002$. 